\title{
APLICAÇÃO DE REJEITOS INDUSTRIAIS NA BIOSSÍNTESE DE CAROTENOIDES POR Rhodotorula mucilaginosa EQTT1
}

\author{
T. A. SCHUELER ${ }^{1}$, V. SOARES ${ }^{2}$, A. J. R. SILVA² e E. F. C. SÉRVULO ${ }^{1}$ \\ 1Universidade Federal do Rio de Janeiro, Departamento de Engenharia Química \\ ${ }^{2}$ Universidade Federal do Rio de Janeiro, Núcleo de Pesquisas de Produtos Naturais \\ E-mail para contato: tamaraschueler@yahoo.com.br
}

\begin{abstract}
RESUMO-Neste estudo foi dada ênfase à obtenção de $\beta$-caroteno, pela estirpe de Rhodotorula mucilaginosa EQTT1. A produção foi realizada tendo como únicas fontes nutricionais a glicerina bruta (GB), oriunda da produção de biodiesel e levedura residual cervejeira (LRC), rejeito da produção de cerveja, visando estabelecer uma alternativa biotecnológica para o aproveitamento dos coprodutos (GB e LRC) gerados em grande quantidade pelas indústrias de origem das matérias primas. Foram testadas diferentes concentrações de carbono e nitrogênio levando em consideração a composição de ambos os rejeitos (GB e LRC).O estudo foi conduzidocom base em planejamento experimental do tipo Doehlert, para duas variáveis, realizados em frascos Erlenmeyer de $500 \mathrm{~mL}$ de capacidade,

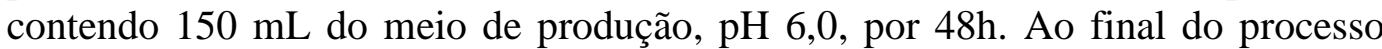
foram determinados: concentraçãode biomassa e de $\beta$-caroteno; e avaliado estatisticamente a melhor condição para maximização da bioprodução do produto em interesse.As diferentes concentrações de nitrogênio e carbono tiveram influência marcante na síntese de $\beta$-caroteno. $O$ valor máximode $\beta$-caroteno $(120,1 \mathrm{mg} / \mathrm{L})$ foiobtido para as concentrações de nitrogênio e de carbono nos pontos máximo e central, respectivamente.Comparando os resultados com dados da literatura, pode-se aferir a possibilidade do emprego desses rejeitos industriais para a obtenção por via microbiana dessebioproduto de grande valor comercial.
\end{abstract}

\section{INTRODUÇÃO}

Os corantes têm sido intensivamente utilizados pelas empresas do setor alimentício para conferir, intensificar, restaurar ou alterar acoloração de alimentos e bebidas, com o propósito de atribuir uma aparência natural aos produtos industrializados, ou, tão somente, torná-los mais atrativos (CONSTANT, STRIGHETA \& SANDI, 2002; ANVISA, 2014).

Em especial, as indústrias alimentícias fazem uso de corantes artificiais, que, dependendo da concentração ingerida, pode acarretar diferentes reações adversas, a curto e em longo prazo, tais como reações alérgicas, ação mutagênica e carcinogênica, anemias e mortalidade de fetos entre outros (ANTUNES \& ARAUUJO, 2000; CARVALHO, 2005; VERÍSSIMO et al., 2008).

No Brasil, o Ministério da Saúde permite o uso de apenas onze corantes artificiais em produtos alimentícios, em concentrações controladas (KAPOOR et al., 2001; PRADO \& GODOY, 2007). Existe um valor de ingestão diária aceitável (IDA) para cada aditivo, estabelecido pela Agencia Nacional de Vigilância Sanitária (PRADO \& GODOY, 2007; 
SCHUMANN, POLÔNIO \& GONÇALVES, 2008). Porém, a falta de uma fiscalização rígida não permite saber se as indústrias estão usando os aditivos nas doses recomendadas para que não seja ultrapassada a IDA.

Neste contexto, surgiu o interesse pelo uso de corantes de origem natural em substituição aos corantes artificiais. Dentre os corantes naturais, os que mais se destacam pertencem ao grupo dos carotenoides, pelas suas propriedades funcionais, já que estão amplamente distribuídos na natureza, podendo ser encontrados em insetos, animais, plantas e microrganismos (UENOJO et al., 2007; RIBEIRO, 2008; TATSCH, 2008). Estes compostos orgânicos proporcionam inúmeros benefícios à saúde humana como: (i) inibição de doenças causadas por radicais livres (SHEKELLE et al., 1981; FONTANA et al., 2000; DONALDSON, 2004; UENOJO et al., 2007; RIBEIRO, 2008; TATSCH, 2008); (ii) efeito antioxidante; (iii) ação anticancerígena (ALMEIDA, 2005).

Dentre os microrganismos produtores de carotenoides, algumas espécies de leveduras são biotecnologicamente interessantes, por sua capacidade de utilização de diferentes substratos (MALDONADE, 2007; TATSCH, 2008), bom rendimento em biomassa, e serem fontes potenciais de proteínas e lipídeos (SQUINA \& MERCADANTE, 2003; MALDONADE, 2003). Em especial, as leveduras do gênero Rhodotorula, por serem capazes de produzir carotenoides a partir de subprodutos industriais, o que minimiza os custos da produção (AKSU \& EREN, 2007; VALDUGA et al., 2007).

A reintrodução de rejeitos na cadeia produtiva tem, como principais vantagens, a redução do custo de produção devido a grande oferta e baixo preço, e a mitigação do seu descarte, evitando danos ao ambiente. Adicionalmente, o aproveitamento de rejeitos é de interesse para as indústrias que os geram, já que diminuindo a quantidade a ser tratada há um decréscimo considerável dos custos operacionais relativos a sua adequação para descarte segundo a legislação.

Este trabalho teve como objetivo geral avaliar a produção de $\beta$-caroteno pela levedura Rhodotorula mucilaginosa EQTT1 em meio formulado apenas com subprodutos industriais glicerina bruta (GB - rejeito da produção do biodiesel) e levedura residual cervejeira (LRC rejeito da produção de cerveja) - como fontes exclusivas de nutrientes, especialmente carbono e nitrogênio.

\section{MATERIAIS E MÉTODOS}

\subsection{Meio de produção}

Foi utilizado um meio líquido constituído exclusivamente de rejeitos oriundos de atividades industriais: levedura residual cervejeira $(400 \mathrm{~g} / \mathrm{kg}$ de proteína bruta) e glicerina bruta $(86,7 \%$ de glicerol), em diferentes proporções. Após ajuste do $\mathrm{pH}$ a 6,0, o meio de cultura foi esterilizado a $121^{\circ} \mathrm{C}$ por 20 minutos.

Os teores de carbono orgânico total (COT) e de nitrogênioforam determinados em analisador Shimadzu, modelo TOC-VCPN (Shimadzu do Brasil, São Paulo, SP). A concentração de carbono e nitrogênio para GB foram 385,6 g/L e 0,037 g/L, já para LRC as concentrações solúveisdos teores analisados foram $0,02 \mathrm{~g} / \mathrm{L}$ e $0,67 \mathrm{~g} / \mathrm{L}$, respectivamente. 


\subsection{Etapas experimentais}

Foram testadas diferentes concentrações de carbono e nitrogênio levando em consideração a composição de ambos os rejeitos usados como únicas fontes nutricionais na formulação do meio de produção.Os ensaios foram conduzidos seguindo o Planejamento do tipo Doehlert para duas variáveis, perfazendo um total de 7 experiências (Figura 1), mais as replicatas do ponto central, totalizando 9 experimentos.

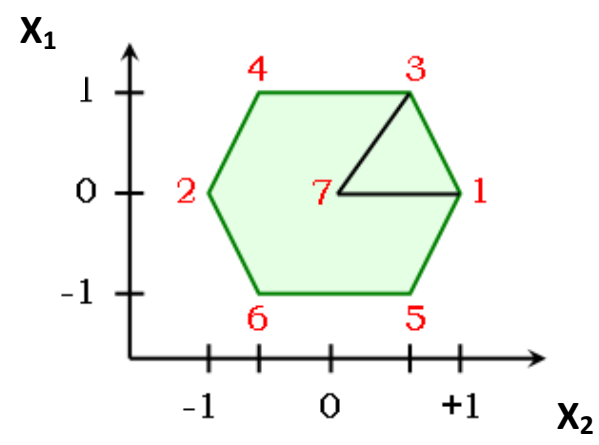

Figura 1:Representação do Planejamento do tipo Doehlert para dois fatores.

As variáveis analisadas foram: concentração de carbono $\left(\mathrm{X}_{1}\right)$ e concentração de nitrogênio $\left(\mathrm{X}_{2}\right)$. Para a concentração de nitrogênio, foi estabelecido para o ponto máximo $(+1)$ o valor de 0,50 $\mathrm{g} / \mathrm{L}$; para o nível inferior (-1) o valor de $0,03 \mathrm{~g} / \mathrm{L}$ e para o ponto central (0) o valor de $0,27 \mathrm{~g} / \mathrm{L}$. Para os pontos intermediários $-0,5$ e $+0,5$ foram dados os valores de 0,15 e $0,38 \mathrm{~g} / \mathrm{L}$, respectivamente. No caso do teor de carbono, foi estabelecido para o nível superior, representado pelo sinal +, o valor de $25 \mathrm{~g} / \mathrm{L}$; para o níveis inferior (-), o valor $5 \mathrm{~g} / \mathrm{L}$; e $15 \mathrm{~g} / \mathrm{L}$ para o ponto central (0).

Para tanto, os experimentos foram realizados em frascos Erlenmeyer de $500 \mathrm{~mL}$ de capacidade contendo $150 \mathrm{~mL}$ do meio de produção e $10^{6}$ células $/ \mathrm{mL}$ da levedura, que foram dispostos em agitador rotatório (Cientec, CT-712RN, Brasil), a $28 \pm 1^{\circ} \mathrm{C}$, sob agitação de $150 \mathrm{rpm}$ (deslocamento do shaker de $60 \mathrm{~mm}$ ) por $48 \mathrm{~h}$, em frascos de sacrifício, na ausência total de luz.Para tanto, os frascos foram recobertos com papel alumínio.

Ao final do processo, foram realizadas as determinações das concentrações de biomassa e de $\beta$-caroteno. Para tanto, os meios fermentados foram centrifugadas a $2332 \mathrm{~g}$ por 10 minutos a $4^{\circ} \mathrm{C}$, para separação das células (BIOVERA, RB7-R, Brasil). Os pellets celulares, resultantes da etapa de centrifugação dos mostos, foram destinados para quantificação de $\beta$-caroteno produzido.

Para a extração dos carotenoides, as células foram primeiramente lisadas em homogeinizador(B. BraunBiotechInternationalHomogenizer, 853023/8, Alemanha), com auxílio de pérolas de vidro, sob atmosfera de $\mathrm{CO}_{2}$, por $1 \mathrm{~min}$. A seguir, as células lisadas foram tratadas com uma mistura de solventes (hexano, acetona e etanol, na proporção 2:1:1), e mantidas em vortex, por 30 s., para a extração total dos carotenoides. 


\subsection{Concentração de biomassa}

A contagem celular foi realizada em microscópio óptico com aumento de 400x, com auxílio da câmara de Neubauer. Com este fim, amostras do meiofermentado foram adequadamente diluídas 1:50 em balão volumétrico e corado com azul de metileno, que cora as células inativas. A concentração de células foi calculada através da equação (1):

$$
\frac{\text { células }}{m L}=\sum \text { células contadas } \times 5 \times 10^{4} \times \text { diluição }^{-1}
$$

\subsection{Caracterização do $\beta$-caroteno e as condições operacionais para a análise cromatográfica}

Para a determinação quantitativa de $\beta$-caroteno, foi considerado o tempo de retenção do padrão de $\beta$-caroteno (Sigma-Aldrich ${ }^{\circledR}$ ).A análise cromatográfica foi feita por CLAE-UV (Agilent Technologies, 1260-Infinity, Alemanha). Foi utilizada a análise em modo gradiente, coluna C30 ( 250 x 4,6 mm; 5 micrometros) (Waters, Irlanda), com temperatura de $20^{\circ} \mathrm{C}$, eluição na vazão de $1 \mathrm{~mL} / \mathrm{min}$., volume de injeção $20 \mu \mathrm{L}$, comprimento de onda $489 \mathrm{~nm}$, tendo metanol/éter metil tercbutílico como fase móvel.

\subsection{Análises estatísticas}

As análises estatísticas foram realizadas no software Excel (Microsoft, 2010).

\section{RESULTADOS E DISCUSSÃO}

\subsection{Concentração celular}

A análise da figura 2 permite inferir que todas as condições nutricionais ensaiadas foram favoráveis ao crescimento da levedura. Portanto, o emprego apenas dos rejeitos LRC e GB atende as necessidades básicas para atividade metabólica da estirpe $R$. mucilaginosa EQTT1, mesmo com variações de relação $\mathrm{C} / \mathrm{N}$ de 15 a $500 \mathrm{~g} / \mathrm{g}$.

As formulações estudadas foram capazes de proporcionar aumento do número de células viáveis em até duas ordens de grandeza. Entretanto, no conto geral, a variação das proporções de LRC e GB não teve influência marcante no crescimento da levedura, uma vez que o número final variou de $5,5 \times 10^{7}$ a $2,1 \times 10^{8}$ células $/ \mathrm{mL}$. 


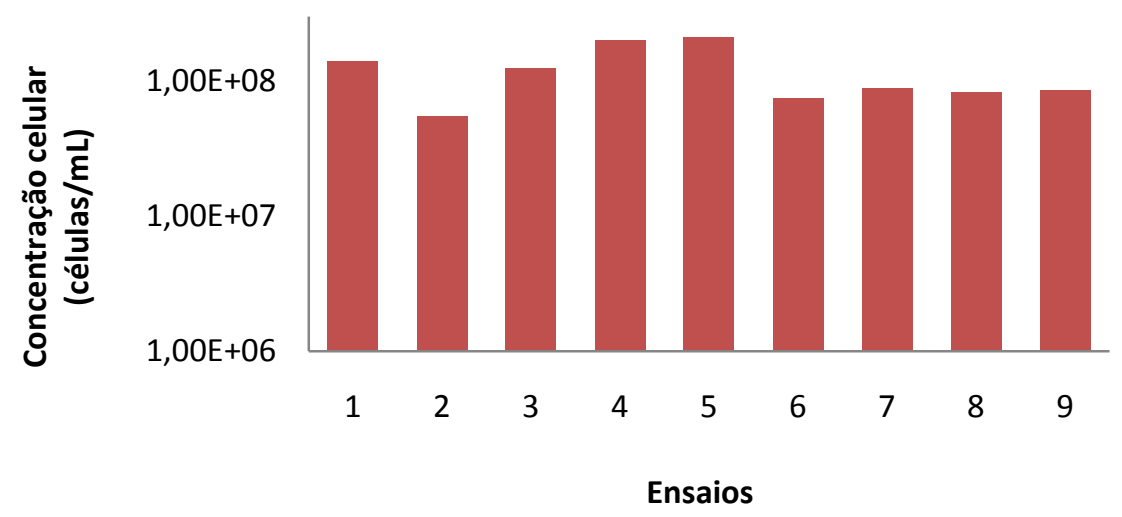

Figura 2 -Variação do número de células durante o cultivo de Rhodotorula mucilaginosa EQTT1 em diferentes concentrações de GB e LRC.

\subsection{Análise estatística da biossíntese de $\beta$-caroteno}

A figura 3 apresenta as concentrações de $\beta$-caroteno, expressas em valores médios de $\mathrm{mg} / \mathrm{L}$, produzidas por $R$. mucilaginosa EQTT1 em meios formulados com distintas proporções de GB e LRC. Conforme pode ser observado, a condição nutricional teve forte influência na síntese microbiana de $\beta$-caroteno, exceto para o ensaio 2 , onde não foi observada produção de pigmentos. O ensaio 1 foi o que propiciou maior produção de $\beta$-caroteno $(120,1 \mathrm{mg} / \mathrm{L})$.

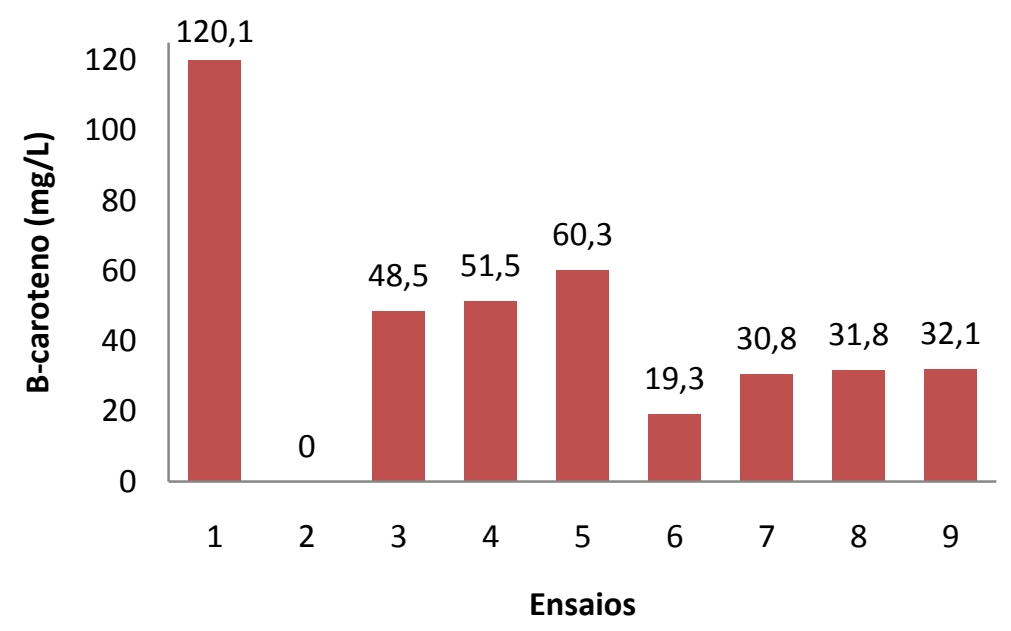

Figura 3 -Biossíntese de $\beta$-caroteno por Rhodotorula mucilaginosa EQTT1.

A metodologia de "superfície de resposta" possibilita definir a tendência das condições para maximização da síntese microbiana de carotenoides e, assim, determinar os valores ideais de carbono e de nitrogênio para produção de pigmentos pela estirpe $R$. mucilaginosa EQTT1 a partir de GB e LRC. A análise das superfícies de resposta plotadas na forma de gráficos 3D (Figura 4) e 2D (Figura 5) para $\beta$-carotenopermite evidenciar o efeito da interação entre as variáveis independentes (GB e LRC). 
Como o planejamento do tipo Doehlert é do tipo esférico, considera-se que os valores das extremidades não são reais, ou seja, são de difícil reprodutibilidade. A observação das respostas dos gráficos (Figuras 4 e 5) demonstra que a variável "concentração de nitrogênio" é a que mais influência na bioprodução de $\beta$-caroteno. Fica evidente que, tomando um nível mais alto de nitrogênio (concentração) e um nível intermediário de carbono (concentração), são obtidos rendimentos mais otimizados (Figura 5). Logo, para maximizar a produção de $\beta$-caroteno seria interessante trabalhar numa concentração central de carbono $(15 \mathrm{~g} / \mathrm{L})$ e numa concentração de nitrogênio em torno da máxima $(0,40 \mathrm{~g} / \mathrm{L})$.

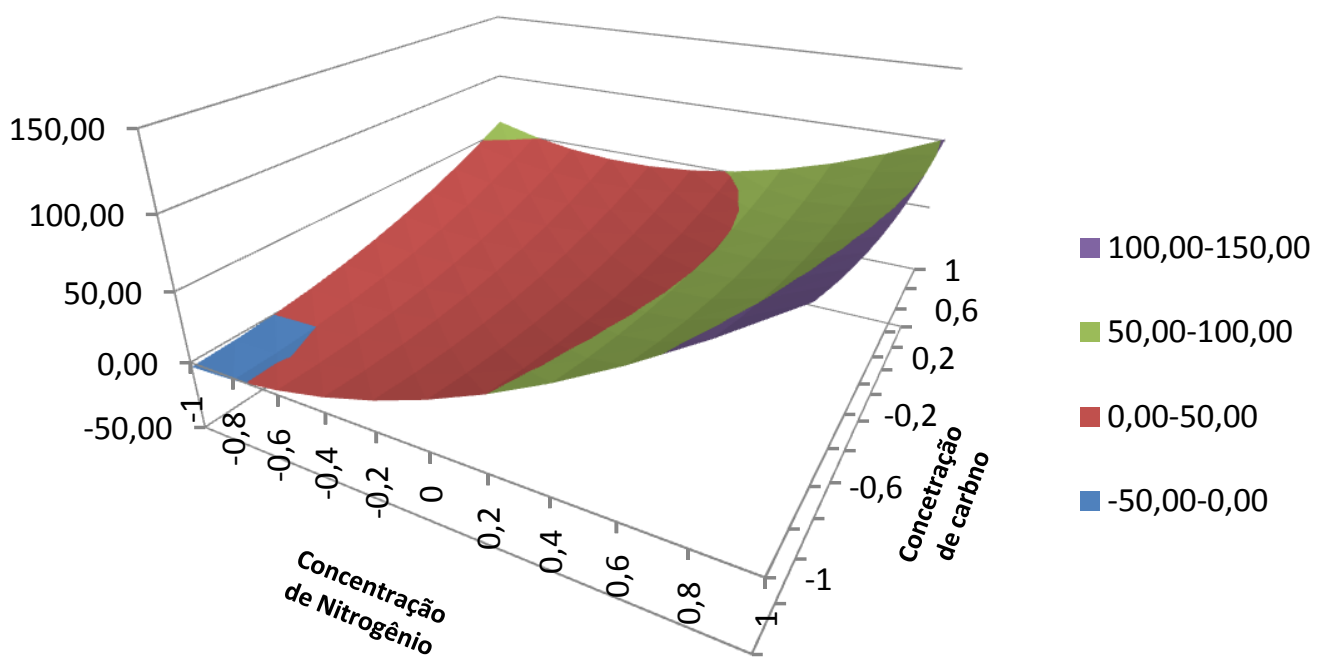

Figura 4 - Superfície de resposta (gráfico 3D) para biossíntese de $\beta$-caroteno $(\mathrm{mg} / \mathrm{L})$ em função da concentração de carbono e da concentração de nitrogênio.
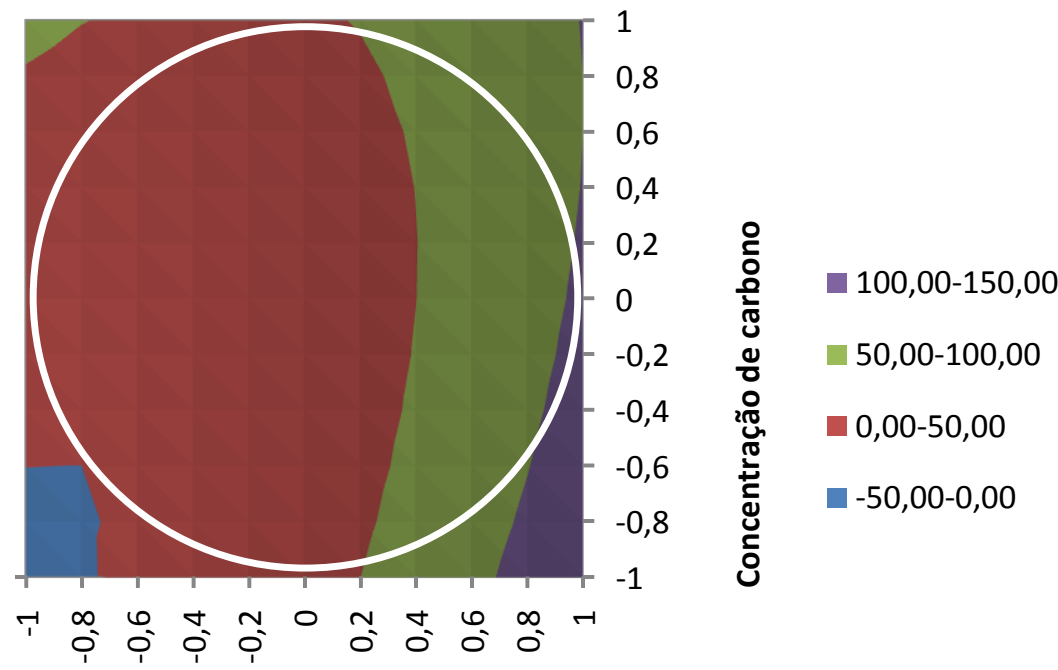

\section{Concentração de nitrogênio}

Figura 5 - Superfície de resposta (gráfico 2D) para biossíntese de $\beta$-caroteno $(\mathrm{mg} / \mathrm{L})$ em função da concentração de carbono e da concentração de nitrogênio. 


\section{CONCLUSÕES}

Os resultados obtidos neste trabalho permitem concluir que:

- É tecnicamente viável o uso de um meio formulado tão somente com os rejeitos industriais, glicerina bruta (GB) e levedura residual cervejeira (LRC), sem qualquer tratamento prévio, para a produção de carotenoides;

- O planejamento do tipo Doehlert se mostrou eficiente para definição dos teores de carbono e de nitrogênio, indicando como condições nutricionais ideais $15 \mathrm{~g} / \mathrm{L}$ de carbono e 0,40 $\mathrm{g} / \mathrm{L}$ de nitrogênio para a biossíntese de $\beta$-caroteno;

- O crescimento celular foi basicamente igual para todos os ensaios, com exceção da condição de maior relação carbono/nitrogênio $(500 \mathrm{~g} / \mathrm{g})$, o que demostra que o crescimento celular independe da produção de carotenoides (metabolismo secundário);

- O máximo de $\beta$-caroteno (120,1 mg/L) foi alcançado em $48 \mathrm{~h}$ de processo na melhor condição avaliada de GB e LRC.

\section{REFERÊNCIAS BIBLIOGRÁFICAS}

AKSU, Z. \& EREN, A. T. Production of carotenoids by the isolated yeast of Rhodotorulaglutinis.Biochemistry Engineering Journal, 35: 107-113, 2007.

ALMEIDA, G.M.D. Rhodotorulaspp. Isolada de hemocultura no hospital das clínicas da faculdade de medicina da Universidade de São Paulo: Características clínicas e microbiológicas. Tese de doutorado em Ciências, da Faculdade de Medicina da Universidade de São Paulo. São Paulo. 2005.

ANTUNES, L. M. G. \& ARAÚJO, M. C. P. Mutagenicidade e antimutagenicidade dos principais corantes para alimentos. Revista Nutrição, 13(2): 81-88, 2000.

ANVISA. Agência Nacional de Vigilância Sanitária. Resolução nº 44/77 de 1977 (DOU 01/02/78). Disponível em: http://www.anvisa.gov.br. Acessado em abril de 2014.

CARVALHO, P. R. Aditivos dos alimentos. Revista Logos, 12: 57-69, 2005.

CONSTANT, P. B.; STRINGHETA, P. C. \& SANDI, D. Corantes alimentícios. Boletim do CEPPA, 2 (20): 203-220, 2002.

DONALDSON, M. S. Nutrition and cancer: A review of the evidence for an anti-cancer diet. Nutritional Journal, 3(19): 1-21, 2004. 


\section{9 a 22 de outubro de 2014 \\ Florianópolis/SC}

FONTANA J. D.; MENDES, S. V.; PERSIKE, D. S.; PERACETTA, L. F. \& PASSOS, M. Carotenoides: cores atraentes e ação biológica. Biotecnologia, Ciência e Desenvolvimento, 13: 4045, 2000.

KAPOOR, M.; BEG, Q.K.; MAHAJAN, L. \& HOONDAL, G.S. Microbial xylanases and their industrial applications: a review. Applied Microbiology Biotechnology, 56: 326 - 338. 2001.

MALDONADE, I. R.; SCAMPARINI, A. R. P. \& AMAYA, D. B. R. Selection and characterization of carotenoid-producing yeasts from campinas region, Brazil. Brazilian Journal of Microbiology, 38: 65-70, 2007.

MALDONADE, I.R. Produção de carotenoides por leveduras. Tese de doutorado em Ciência de Alimentos da UNICAMP. Campinas. 2003.

PRADO, M. A. \& GODOY, H. T. Teores de corantes artificiais em alimentos determinados por cromatografia líquida de alta eficiência. Química Nova, 2 (30): 268-273, 2007.

RIBEIRO, B. D. Aplicação de tecnologia enzimática na obtenção de $\beta$-caroteno a partir de óleo de buriti (MAURITIA VINIFERA). 2008. 103 f. Dissertação de Mestrado em Tecnologia de Processos Químico e Bioquímicos, UFRJ, Rio de Janeiro, 2008.

SCHUMANN, S. P. A.; POLÔNIO, M. L. T \& GONÇALVES, E. C. B. A. Avaliação de consumo de corantes artificiais por lactantes, pré-escolares e escolares. Ciência e Tecnologia de Alimentos, 28(3). 2008.

SHEKELLE, R. B.; LIU, S.; RAYNOR, W. J. J.; LEPPER, M.; MALIZA, C.; ROSSOF, A. H.; PAUL, O.;SHRYOCK, A. M. \& STAMLER, J. Dietary vitamin A and risk of cancer in the western electric study. The Lancet Ltd, 1981.

SQUINA, F. M. \& MERCADANTE, A. Z. Análise, por CLAE, de carotenoides de cinco linhagens de Rhodotorula. Revista Brasileira de Ciências Farmacêuticas, 3(39): 309 - 318, 2003.

TATSCH, P.O. Produção de carotenoides em meio sintético por SporidiobolussalmonicolorCBS 2636 em biorreator. 2008. 94 f. Dissertação de Mestrado em Engenharia de Alimentos do Programa de Pós-Graduação em Engenharia de Alimentos, Universidade Regional Integrada do Alto Uruguai e das Missões, Rio Grande do Sul, 2008.

UENOJO, M.; MARÓSTICA, M. R. J \& PASTORE, G. M. Carotenoides: Propriedades, aplicações e biotransformação para a formação de compostos de aroma. Química Nova, 3(30): 616-622, 2007.

VALDUGA, E.; VALERIO, A.; TREICHEL, H. \& LUCCIO, M. Pré-tratamento de melaço de cana-de-açúcar e água de maceração de milho para a bioprodução de carotenoides. Quimíca Nova, 30(8): 1860-1866, 2007.

VERÍSSIMO, S. A; OLIVEIRA, E. L.; LADCHUMANANANDASIVAM, R.; AQUINO, M. S. \& ALEXANDRE, M. E. O. Aproveitamento do corante natural (Bixaorellana) no tingimento de fibra celulósica. Tecnologia \& Ciência Agropecuária, 2: 35-39, 2008. 